\title{
Avaliação físico-química do óleo extraído de amendoim (arachis hypogaea)
}

\author{
Physical and chemical evaluation of extracted peanut oil (arachis hypogaea) \\ Evaluación física y química del aceite de maní extraído (arachis hypogaea)
}

Recebido: 15/01/2021 | Revisado: 18/01/2021 | Aceito: 21/01/2021 | Publicado: 25/01/2021

Auanna Marcelly Soares de Oliveira
ORCID: https://orcid.org/0000-0002-0281-3721
Instituto Federal de Educação, Ciência e Tecnologia do Piauí, Brasil
E-mail: auannamarce06@gmail.com
Juliana Chaves Elias Santos
ORCID: https://orcid.org/0000-0002-6307-0183
E-mail: julliana-c-e@ @otmail.com
Edilene Ferreira da Silva
Instituto Federal de Educação, Ciência e Tenologia do Piaú Brat
ORCID: https://orcid.org/0000-0001-7073-7962
Universidade Federal de Santa Catarina, Brasil
E-mail: silvaedilene16@ @otmail.com
Victória Maura Silva Bermúdez
ORCID: https://orcid.org/0000-0002-8105-8735
Universidade Federal do Ceará, Brasil
E-mail: vmsbermudez@gmail.com
Vera Lúcia Viana do Nascimento
ORCID: https://orcid.org/0000-0003-0071-3863
E-mail: veravnascimento@gmail.com

\section{Resumo}

O óleo extraído da semente de amendoim tem propriedades benéficas á saúde por ser antioxidante, antinflamatório e prevenir doenças coronárias. Apesar desta qualidade, o processamento e armazenamento inadequado pode causar degradações como as oxidações lipídicas devida sua poli-insaturação. O trabalho teve o objetivo de avaliar as características físico-químicas do óleo extraído de amendoim e do óleo comercial de amendoim. A extração e análises do índice de acidez, \% de Ácidos Graxos Livres (AGL), índice de peróxido e índice de iodo do óleo foram realizadas nos laboratórios do Instituto Federal do Piauí (IFPI), campus Teresina Central. O óleo de amendoim foi extraído com hexano, pelo método Bligh \& Dyer e o óleo de amendoim comercializado foi obtido no mercado local. O óleo extraído pelos dois métodos estava com os índices óleoquímicos dentro do preconizado pela legislação enquanto o óleo industrializado estava acima do padrão. O método de extração de maior rendimento foi Bligh \& Dyer, demonstrando que o método de extração interfere nas características físico-químicas e rendimento do óleo.

Palavras-chave: Controle de qualidade; Extração por solvente; Oleaginosa; Óleo vegetal; Poli-insaturados.

\begin{abstract}
The oil extracted from the peanut seed has beneficial health properties as it is antioxidant, anti-inflammatory and prevents coronary heart disease. Despite this quality, improper processing and storage can cause degradation such as lipid oxidation due to its polyunsaturation. The work aimed to evaluate the physical and chemical characteristics of oil extracted from peanuts and commercial peanut oil. The extraction and analysis of the acidity index, \% of Free Fatty Acids (AGL), peroxide index and oil iodine index were carried out in the laboratories of the Federal Institute of Piauí (IFPI), Teresina Central campus. The peanut oil was extracted with hexane, using the Bligh \& Dyer method and the commercialized peanut oil was obtained in the local market. The oil extracted by the two methods had the oil-chemical indexes within that recommended by the legislation while the industrialized oil was above the standard. The most efficient extraction method was Bligh \& Dyer, demonstrating that the extraction method interferes with the physicochemical characteristics and oil yield.
\end{abstract}

Keywords: Oilseed; Vegetable oil; Solvent extraction; Quality control; Polyunsaturated.

\section{Resumen}

El aceite extraído de la semilla de maní tiene propiedades beneficiosas para la salud, ya que es antioxidante, antiinflamatorio y previene la enfermedad coronaria. A pesar de esta calidad, un procesamiento y almacenamiento incorrectos pueden provocar degradaciones como la oxidación de los lípidos debido a su poliinsaturación. El trabajo tuvo como objetivo evaluar las características físicas y químicas del aceite extraído del maní y del aceite de maní comercial. La extracción y análisis del índice de acidez, \% de ácidos grasos libres (AGL), índice de peróxidos e índice de yodo en aceite se realizaron en los laboratorios del Instituto Federal de Piauí (IFPI), campus Teresina Central. El 
aceite de maní se extrajo con hexano, mediante el método Bligh \& Dyer y el aceite de maní comercializado se obtuvo en el mercado local. El aceite extraído por los dos métodos tenía los índices aceite-químicos dentro de los recomendados por la legislación mientras que el aceite industrializado estaba por encima del estándar. El método de extracción más eficiente fue Bligh \& Dyer, demostrando que el método de extracción interfiere con las características fisicoquímicas y el rendimiento de aceite.

Palabras clave: Aceite vegetal; Control de calidad; Extracción por solvente; Oleaginoso; Poliinsaturado.

\section{Introdução}

O amendoim (Arachis Hypogaea) são sementes comestíveis de uma leguminosa com excelentes propriedades nutricionais, sendo que seus grãos contêm grande quantidade de proteínas e lipídios, possuindo ainda uma boa fonte de minerais como, fósforo, cálcio, magnésio e potássio (Nunes, 2018). É rica em compostos antioxidantes como resveratrol e ácidos fenólicos e outros nutrientes com propriedades funcionais como flavonóides e fitoesteróis, que desempenham, dentre outras funções, proteção das células contra os danos causados pelo estresse oxidativos, raios solares, dentre outros fatores, demonstrando uma boa capacidade antioxidativa e antinflamatória (Ma et al., 2014).

A Arachis Hypogaea contém uma alta proporção de ácidos graxos mono e poli-insaturados, característica desejável por reduzir a quantidade de colesterol no sangue, bem como a baixa densidade e conteúdo de lipoproteínas, o que pode reduzir o risco de doença cardíaca coronária (Win et al., 2011). A composição destes ácidos graxos influencia na qualidade nutricional e no armazenamento de amendoim, fatores importantes que contribuem na melhoria da saúde dos consumidores (Shasidhar et al., 2017).

O Brasil apresenta-se como grande produtor dessa oleaginosa. Na região Sudeste o Estado de São Paulo é o maior produtor de amendoim, sendo responsável por $80 \%$ do que é produzido no Brasil. No entanto, a região Nordeste é o segundo maior polo consumidor no país, uma média de 50 mil toneladas por ano (EMBRAPA, 2014).

Apesar das características dessa oleaginosa e do seu óleo extraído, diversos fatores podem afetar sua estabilidade lipídica, pois são ricas em acidos graxos poli-isaturados como longos períodos de armazenamento, comprometendo sua qualidade e conservação, onde várias reações lipídicas de decomposição ocorrem. Existem diferentes mecanismos para que um óleo seja oxidado. A autoxidação e a oxidação por fotossensibilidade são duas formas desta alteração lipídica ocorrer, sendo que esta uma reação apenas com ácidos graxos insaturados, estando livres ou esterificados como molécula de triglicerídeo e oxigênio (Almeida et al., 2019). Devido a isto, torna-se necessária a conservação do produto para evitar alterações de degradações lipídicas, uma vez que a avaliação da qualidade do óleo é realizada por vários testes oleoquímicos, que identificam os processos oxidativos nos alimentos.

A estabilidade oxidativa é um dos principais indicadores da qualidade do óleo vegetal se encontra (Tan et al., 2017). Esta depende de vários fatores como temperatura, luz, oxigênio, metais, enzimas, presença de antioxidantes ou pro-oxidantes, composição de ácidos graxos do óleo e uso de embalagens que permitam entrada de oxigênio (Ahmad et al., 2011; Pristouri et al., 2010).

Diante do exposto, as características dos óleos vegetais podem apresentar alterações lipídicas pela influência de diversos fatores físicos e químicos, como temperatura, luz, oxigênio singleto e os antioxidantes, que influenciam na composição das gorduras do ponto de vista do grau de insaturação. Então, pois os óleos vegetais de amendoim são poli-insaturados, por isso instáveis com reação às duplas ligações químicas, sendo necessário o controle de qualidade das matérias primas ideais para consumo e fornecimento dos benefícios da saúde humana.

Este trabalho teve o objetivo de avaliar as características físico-químicas do óleo extraído e comercial de amendoim (arachis hypogaea), por meio de análises químicas de controle de qualidade específicas para óleos, com base em parâmetros de identificação da acidez, ácido graxos livres, formação de peróxidos e detecção do grau de insaturação do óleo vegetal por determinação do iodo, todos importantes para conservação, processamento e armazenamentos dos alimentos. 


\section{Metodologia}

A pesquisa é de caráter experimental (Pereira et al., 2018) para a realização de índices, estatísticas e porcentagens. As análises foram realizadas no laboratório de Tecnologia de produtos de origem vegetal (TPOV), Bromatologia, Laboratório de Química Analítica, Inorgânica e físico-química do Departamento de química, do Instituto Federal do Piauí (IFPI), campus Teresina central.

\subsection{Aquisição da matéria-prima}

As amostras de amendoim com cascas (Figura 1) foram adquiridos no mercado local, as amostras de amendoim foram acondicionadas adequadamente e conduzidas ao laboratório. O óleo de amendoim industrializado (Figura 2) foi adquirido na loja Zona Cerealista Online e armazenado em frasco pequeno de cor âmbar.

Figura 1 - Amendoim in natura.

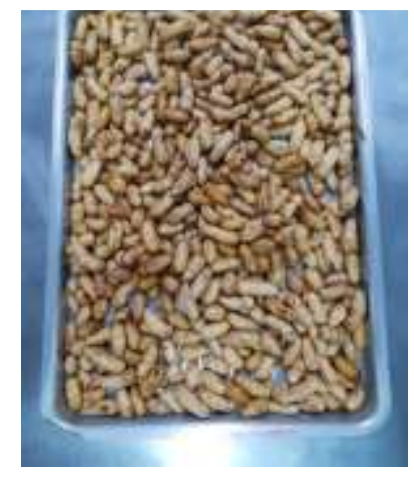

Fonte: Autores (2021).
Figura 2 - Óleo de amendoim industrializado.

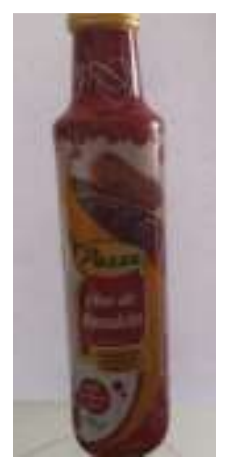

Fonte: Autores (2021).

\subsection{Delineamento experimental}

O planejamento experimental foi do tipo casualizado, onde as amostras de óleo de amendoim (Arachis Hypogaea) foram analisadas em triplicatas. Foram 03 tratamentos, 06 análises, 3 repetições, totalizando 45 amostras.

As amostras foram analisadas pelos índices oleoquímicos, conforme o Official methods and recommended practices of the American Oil Chemists' Society (AOCS, 2004), que incluíram os seguintes índices: Índices de Acidez (IA), Índice de Ácidos Graxos Livres (\% AGL), Índice de Peróxido (IP). Índice de Iodo (II).

\subsection{Extração do óleo de amendoim (arachis hypogaea)}

Os amendoins com casca foram selecionados, excluindo os amendoins com presença de manchas escuras, posteriormente, foram pré-tratados, triturados para obtenção da farinha para extração do óleo.

\subsection{Preparação das sementes}

As amostras foram lavadas em água corrente, higienizadas em água hiperclorada a $20 \mathrm{ppm}$, após isso, foram submetidas a secagem em estufa a temperatura de $50{ }^{\circ} \mathrm{C}$ por 60 minutos. Em seguida, foram descascadas manualmente e colocadas em estufa a $45{ }^{\circ} \mathrm{C}$, por 60 minutos. Retiradas as amostras da estufa, foram resfriadas em temperatura ambiente e 
posteriormente, retiradas as películas aderidas a semente. As amostras tratadas foram armazenadas em frasco âmbar com tampas vedadas, sob temperatura de refrigeração, a $4^{\circ} \mathrm{C} \pm 2^{\circ} \mathrm{C}$.

\subsection{Extração do óleo de amendoim pelo método Bligh \& Dyer}

Inicialmente, as sementes de amendoim (Arachis Hypogaea) serão lavadas com água destilada e secadas no desidratador, e aerador de alimentos Pratic Dyer a $45^{\circ} \mathrm{C}$ por 2 horas. Em seguida as sementes serão trituradas para obtenção da farinha em triturador industrial Arno, peneiradas e pesadas em balança semi-analítica Bel Engineering. Serão utilizados 100g da farinha, esta quantidade será colocada em béquer de 2 L. Neste, juntamente com a farinha obtida, serão adicionados $400 \mathrm{~mL}$ de metanol, $100 \mathrm{~mL}$ de clorofórmio, e $200 \mathrm{~mL}$ de água destilada, procedimento realizado em uma capela.

Ficará em refrigerador Carel Easy, por $48 \mathrm{~h}$ em temperatura de $10 \pm 2^{\circ} \mathrm{C}$. Em seguida será filtrado com auxílio de um funil, e colocado no decantador de $2 \mathrm{~L}$, sendo adicionado mais $30 \mathrm{~mL}$ de clorofórmio e $40 \mathrm{~mL}$ de água destilada, e deixado em repouso por $24 \mathrm{~h}$.

Após a decantação por 24 horas, será realizada a separação de fases, ficando somente a parte oleosa que será retirada e colocada no banho maria, a $64^{\circ} \mathrm{C}$ por 1 hora e 30 minutos. Posteriormente, foi adicionado uma quantidade suficiente de sulfato de sódio $\left(\mathrm{Na}_{2} \mathrm{~S}_{4}\right)$, para retirada do excesso de reagente, e acomodado o óleo em uma embalagem de vidro âmbar de $250 \mathrm{~mL}$, e armazenado sob refrigeração a $10 \pm 2^{\circ} \mathrm{C}$.

Após passar por todos estes processos, o óleo de amendoim será submetido à rotaevaporação no equipamento Fisatom a temperatura de $50 \pm 2^{\circ} \mathrm{C}$, com banho ultratermostático estabilizado a $15^{\circ} \mathrm{C}$, rotação de $80 \mathrm{rpm}$, vácuo de $-500 \mathrm{mmHg}$ (milímetros de Mercúrio) por 1 h, para retirada de reagentes residuais existentes. Posteriormente, o óleo obtido será armazenado sob refrigeração a $4 \pm 2^{\circ} \mathrm{C}$.

\subsection{Extração do óleo por de solvente}

Inicialmente, as amostras com casca foram lavadas com água destilada para higienização e retirada das impurezas. Após esse pré-tratamento, as amostras foram colocadas em um desidratador PraticDyer a $60 \pm 2{ }^{\circ} \mathrm{C}$ de secagem aerada, para branqueamento das sementes por um período de 2 horas. Após o tempo estabelecido, 200g da amostra foi triturada em liquidificador industrial, peneirada, formando uma farinha de amendoim (Figura 3) e em seguida feita a pesagem em um Becker. Após os procedimentos descritos foi feita a adição inicial de $300 \mathrm{~mL}$ de hexano P.A, misturou-se o solvente juntamente com a amostra com ajuda de um bastão de vidro, depois foi feita a adição de mais $400 \mathrm{~mL}$ do solvente, totalizando $700 \mathrm{~mL}$.

Após um dia em repouso a amostra com o solvente foi colocado em um decantador volume 2 L para a separação das fases, conforme Figura 4, em que se tem a fase líquida separada da fase oleosa. Posteriormente, a separação das fases oleosa e fase líquida, a fase oleosa foi rotaevaporada no equipamento Fisatom (Figura 5) a temperatura de $50 \pm 2{ }^{\circ} \mathrm{C}$, a uma rotação de 80 rpm, sob pressão de $-400 \mathrm{mmHg}$ e com banho ultratermostático estabilizado á $7^{\circ} \mathrm{C}$ por $1 \mathrm{~h}$, para retirada de reagentes que ainda estavam presentes no óleo (Figura 6). Posteriormente, o óleo obtido foi armazenado sob refrigeração á $4 \pm 2{ }^{\circ} \mathrm{C}$ (Figura 7 ). 
Research, Society and Development, v. 10, n. 1, e49710112011, 2021

(CC BY 4.0) | ISSN 2525-3409 | DOI: http://dx.doi.org/10.33448/rsd-v10i1.12011

Figura 3 - Farinha de amendoim.

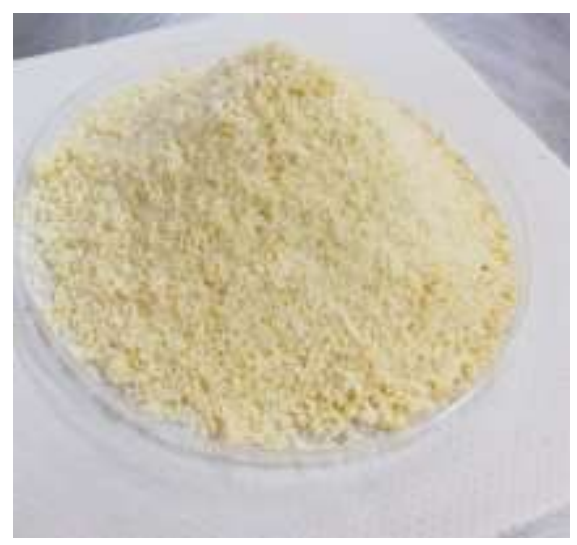

Fonte: Autores (2021).
Figura 4 - Óleo de amendoim em decantador.

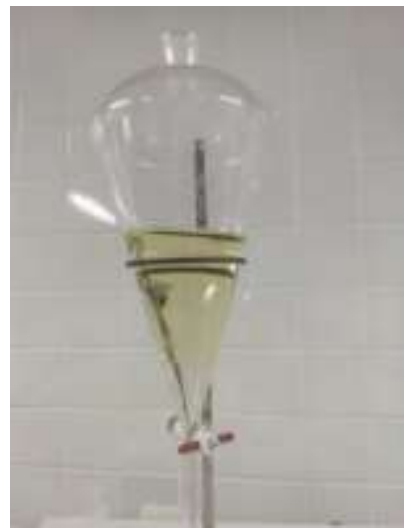

Fonte: Autores (2021).
Figura 5 - Rotaevaporador

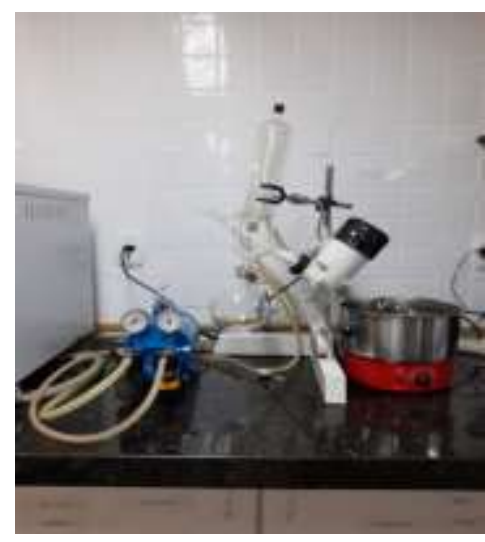

Fonte: Autores (2021).
Figura 6 - Óleo de amendoim extraído por Bligh

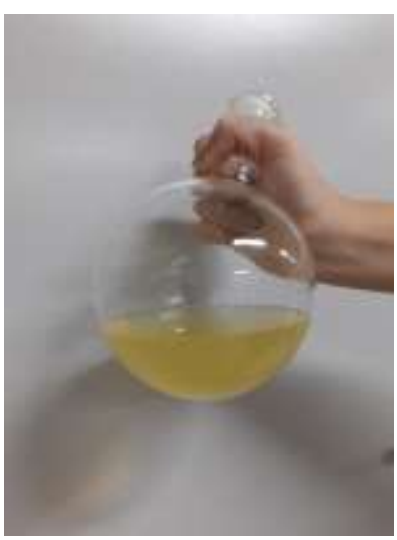

Fonte: Autores (2021).
Figura 7 - Óleo extraído por hexano.

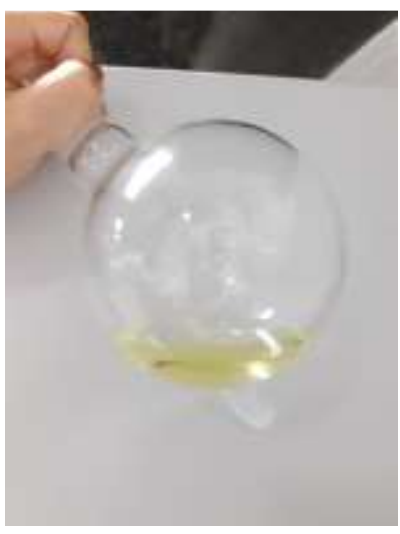

Fonte: Autores (2021).

\subsection{Análises físico-químicas do óleo vegetal}

O óleo de amendoim bruto foi caracterizado por meio de parâmetros físico-químicos (Tabela 1), de acordo com AOCS: Índice de acidez, de ácidos graxos livres, índice de peroxido e índice de iodo. 
Tabela 1 - Parâmetros físico-químicos a serem analisados no óleo.

\begin{tabular}{ccc}
\hline PARÂMETROS & UNIDADE & MÉTODO \\
\hline Acidez & mgNaOH/g & AOCS cd $1 \mathrm{c}-85$ \\
$\%$ Ácidos Graxos Livres & $\mathrm{mg} \mathrm{de} \mathrm{ácido} \mathrm{oléico/100} \mathrm{mg}$ & AOCS Ca $5 \mathrm{a}-406$ \\
Índice de Peróxido & $\mathrm{mEq} \mathrm{O} / \mathrm{kg}$ & AOCS Cd $8-53$ \\
Índice de iodo & $\mathrm{g} \mathrm{I} / 100 \mathrm{~g}$ & AOCS $1 \mathrm{~b}-87$
\end{tabular}

Fonte: Autores (2021).

\subsection{Análise estatística}

As análises estatísticas dos resultados dos parâmetros físico-químicos foram obtidas por meio de média \pm SD (desvio padrão), além da análise de variância (ANOVA) e comparação pelo Teste de Tukey. A significância estatística foi estabelecida em 5\%. Os programas utilizados para realizar essa etapa foram Microsoft Excel e Minitab 17 Statistical Software (2016)

\section{Resultados e Discussão}

As análises físico-químicas do óleo extraído de amendoim (Arachis Hypogaea) com base na metodologia AOCS, e do óleo industrializado de amendoim adquirido no comércio, encontram-se apresentadas na Tabela 2.

Tabela 2 - Valores médios analisados para as determinações dos índices de acidez, \% ácidos graxos livres (\% AGL), peróxido, e de iodo em amostras de óleo de amendoim industrializado, óleo de extraído pelo método Bligh \& Dyer e óleo de amendoim extraído com hexano.

\begin{tabular}{lccc}
\hline \multicolumn{1}{c}{ PARÂMETROS } & NDUSTRIALIZADO & Bligh \& Dyer & HEXANO \\
\hline I.A (mg NaOH/g) & $6,20 \pm 0,20^{\mathrm{a}}$ & $1,17 \pm 0,16^{\mathrm{b}}$ & $0,58 \pm 0,003^{\mathrm{c}}$ \\
\% AGL (mg AG/100mg) & $4,37 \pm 0,14^{\mathrm{a}}$ & $0,82 \pm 0,11^{\mathrm{b}}$ & $0,41 \pm 0,002^{\mathrm{c}}$ \\
I.P (mEq/Kg) & $9,49 \pm 2,73^{\mathrm{a}}$ & $5,09 \pm 2,05^{\mathrm{a}}$ & $6,52 \pm 3,11^{\mathrm{a}}$ \\
I.I (g I/ $\mathbf{~ 1 0 0 ~ g ) ~}$ & $226,00 \pm 45,3^{\mathrm{a}}$ & $200,92 \pm 37,5^{\mathrm{a}}$ & $177,82 \pm 1,54^{\mathrm{a}}$ \\
& & & \\
\hline
\end{tabular}

Índices de Acidez (IA), Índice de Ácidos Graxos Livres (\% AGL), Índice de Peróxido (IP). Índice de Iodo (II). Médias que não compartilham uma letra na mesma linha são significativamente diferentes. Fonte: Autores (2021).

De acordo com o presente estudo, o índice de acidez e o percentual de ácidos graxos livres do óleo de amendoim diferiram estatisticamente $(\mathrm{p}<0,05 \%)$ entre as formas de extração com os diferentes solventes e no óleo industrializado. Por 
outro lado, os valores médios dos índices de peróxido e iodo não diferiram estatisticamente $(\mathrm{p}<0,05 \%)$ entre as formas de extração nem diferiram no óleo industrializado.

O óleo industrializado revelou índice de acidez muito superior aos outros óleos de extração vegetal. De acordo com a Agência Nacional de Vigilância Sanitária (ANVISA), óleos vegetais não refinados, como o caso de todos os óleos de amendoim analisados, não devem ultrapassar acidez de 4,0 mg KOH/g. Portanto, o único óleo de amendoim que se demonstrou acima do padrão exigido pela legislação nacional para acidez foi o óleo industrializado (BRASIL, 2005). O valor de acidez dos óleos extraídos neste trabalho experimental foi considerado muito menor que o encontrado nos dados fornecidos pela literatura. Um estudo encontrou o valor de 4,88 $\mathrm{mg} \mathrm{KOH} / \mathrm{g}$ no óleo de amendoim analisado. O mesmo autor justifica o valor elevado por exposição excessiva à luz e ao armazenamento inadequado. Portanto, demonstrando que o óleo industrializado analisado neste estudo se encontrava em estado de deterioração por falha em seu armazenamento (Radünz et al., 2018). O óleo armazenado em temperaturas inadequadas e na presença de luz pode alterar as características, oxidando mais rapidamente, caso não tenha adição dos antioxidantes.

O valor médio para o percentual de ácidos graxos livres no óleo industrializado foi mais elevado em relação aos outros valores dos óleos vegetais obtidos pela extração com os solventes. O óleo de Pachira aquatica Aublet, por exemplo, apresentou um percentual de 39,2\% de ácidos graxos livres. O autor daquele estudo atribuiu o alto valor de ácidos graxos livres às ações enzimáticas de hidrólises das lipases sobre os triacilgliceróis (Jorge e Luzia, 2012). A acidez livre, portanto, é um parâmetro relacionado com a natureza e qualidade da matéria-prima, com a qualidade e o grau de pureza do óleo e com o processo de extração e conservação do mesmo (Moretto et al., 2002). Em nossos estudos isso pode estar associado à eficiência dos solventes, verificando-se que o hexano extraiu menos.

Os valores médios dos índices de peróxido dos três tipos de óleo de amendoim analisados não diferiram entre si, encontram-se muito abaixo do valor limite permitido pela legislação para óleos vegetais. De acordo com a legislação da ANVISA, os óleos vegetais não refinados não devem ultrapassar de $15 \mathrm{meq} / \mathrm{kg}$ (BRASIL, 2005). Outros autores encontraram valores médios mais elevados (10,17 e 14,57 meq kg-1) (Roberto et al., 2016). Isto demonstra que o óleo extraído e o óleo industrializado apresentaram-se mais estáveis, do ponto de vista da degradação oxidativa devido aos dados dos ácidos graxos lives e seus índices de peróxidos. Pois, elevada quantidade de peróxidos formados indicaria deterioração do produto (De Barros, 2015).

Pighinelli et al. (2008), estudando a "otimização da prensagem a frio de grãos de amendoim em prensa contínua tipo expeller "encontrou valor médio menor ainda (1,20 meq.kg-1 ) para o índice de peróxido em óleo bruto de amendoim, indicando boa qualidade e bom estado de conservação.

Comparando os valores médios dos índices de iodo do óleo de amendoim foram verificados que não diferiram estatisticamente ( $\mathrm{p}<0,05 \%)$. Segundo a CODEX ALIMENTARIUS (2015), o índice de iodo em óleo bruto de amendoim, deve ficar numa faixa de 86-107 g I $/ 2 / 100 \mathrm{~g}$ (Valor de referência da AOCS). Em comparação com as pesquisas existentes. Neste estudo os óleos analisados estavam bem acima $\left(177,82\right.$ a 226,00 g $\left.\mathrm{I}_{2} / 100 \mathrm{~g}\right)$ do resultado de outros autores, que também verificaram que também analisaram o índice de iodo. Outro estudo quantificou 86,7 a 90,9 de índice de iodo em óleos de diferentes espécies de amendoim. Este mesmo trabalho explica que o índice de iodo é importante, por definir o grau de instauração e da instabilidade de óleos. Quanto maior o índice de iodo, maior o grau de insaturação das moléculas lipídicas. Portanto, o óleo de amendoim apresentou-se como bastante insaturação em seus ácidos graxos (Zahran, 2019), consequentemente, consequentemente, exige mais cuidados de conservação para reduzir seus processos oxidativos. Dean et al. (2009) encontraram valores de iodo de 84,1 a 100,3 g I $/ 100 \mathrm{~g}$ ) em 108 linhas de uma coleção de germoplasma nos Estados Unidos, sendo esses valores médios semelhantes a este estudo. 
A Tabela 3, abaixo, mostra o rendimento de óleo pelos métodos de extração Bligh \& Dyer e utilizando solvente hexano para realização da pesquisa.

Tabela 3 - Rendimento de óleo pelos dois métodos de extração realizados.

\begin{tabular}{lcc}
\hline Método de extração & $\begin{array}{c}\text { Quantidade de farinha } \\
\text { utilizada }(\mathbf{g})\end{array}$ & $\begin{array}{c}\text { Quantidade de óleo obtido } \\
(\mathbf{m L})\end{array}$ \\
\hline Hexano & 100 & 13,75 \\
Bligh \& Dyer & 100 & 141,5 \\
\hline
\end{tabular}

Fonte: Autores (2021).

O rendimento de óleo extraído com uso de solvente hexano foi muito menor $(13,75 \%)$ que o óleo obtido pelo método Bligh \& Dyer que foi de 141,5\%. O resultado obtido de porcentagem de óleo foi próximo de outros estudos utilizando hexano. Ao extrair óleo de gérmen de trigo com este mesmo solvente, Ansolin et al., (2013) obteve aproximadamente 10\% de óleo. Vários estudos têm sido publicados sobre como os diferentes solventes de extração impactam a qualidade do óleo, especialen, especialmente, o rendimento do óleo e os níveis de compostos bioativos (Oladipo e Betiku, 2019, Stevanato e da Silva, 2019).

O método de extração Bligh \& Dyer, mostrou-se muito eficiente para extrair outros óleos vegetais de acordo com a literatura, alcançando rendimento de 36,34\% ao extrair óleo de Pinhão Manso (Bento et al., 2017). O alto rendimento de óleo pelo método Bligh \& Dyer é justificado pelo fato de que por meio dessas técnicas é possível extrair todas as classes de lipídios das sementes (Gusso et al., 2012). A produção de óleos a partir de sementes oleaginosas específicas representa desenvolvimento sócio-econômico para o país.

\section{Conclusão}

Neste estudo, concluímos que o óleo extraído de amendoim apresentou as características físico-químicas melhoradas do ponto de vista da qualidade em relação aos índices oleoquímicos avaliados com a extração do óleo por solventes, sendo o método Bligh \& Dyer considerado o mais eficiente que o solvente hexano. Ao contrário, o óleo industrializado foi observado com mais degradações oxidativas, atribuindo-se às influências dos fatores intrínsecos do processamento, da conservação e do armazenamento do produto. Entretanto, outros fatores podem interferir na determinação do material graxo extraído do óleo de amendoim, inclusive, a própria quantidade do rendimento, associada ao tipo de variedade do amendoim.

Podemos enfatizar que mais pesquisas futuras serão necessárias para maiores informações científicas acerca dos fatores que interferem na quantidade e características de qualidade dos óleos vegetais extraídos das variedades especificadas de sementes oleaginosas. Pois, os diferentes métodos de extração, bem como os tipos de solventes otimizados servirão para melhorar o desempenho da produção oleosa, que é importante na aplicação dos processos industriais de matérias primas utilizadas com finalidades comerciais. Mais parâmetros de avaliação servirão para redefinir a qualidade dos óleos vegetais, devido à sua vulnerabilidade ambiental.

\section{Agradecimentos}

Ao Laboratório de Química Analítica, Inorgânica e físico-química do Departamento de química, do Instituto Federal do Piauí (IFPI), campus Teresina central.

\section{Referências}


Ahmad, T., Atta, S., Zeb, A., Gul, S. (2011) Effect of saturation and micro nutritional status on stability of dietary oils under photooxidative stress condition. Journal of the Chemical Society of Pakistan, 33 (3), 343-350.

Association of Official Analytical Chemists - A.O.A.C. (2004) Official Methodos of Analysis of the Association of Analytical Chemists. 13 Ed. Arlington, p. $211-17$.

Almeida, D. T. D., Viana, T. V., Costa, M. M., Silva, C. D. S., Feitosa, S. (2019) Effects of different storage conditions on the oxidative stability of crude and refined palm oil, olein and stearin (Elaeis guineensis). Food Science and Technology, v. 39 (1), 211-217.

Ansolin, M., Basso, R. C., Meirelles, A. J. A., Caldas, E. A. (2013) Experimental data for liquid-liquid equilibrium of fatty systems with emphasis on the distribution of tocopherols and tocotrienols, Fluid Phase Equilibria, 338, 78-86,

Bento, J. A., Silva, M. O., Silva, N. P., Gonçalves, M. A., Evangelista, A. W., De Moura, C. J., Nogueira, R. G. (2017) Avaliação das Metodologias de Prensagem a Frio, Soxhlet e Bligh Dyer, na Extração do Óleo de Pinhão Manso. Revista Processos Químicos, 11 (21), 47-50.

BRASIL. Resolução RDC n ${ }^{\circ}$ 270, de 22 de setembro de 2005. Regulamento técnico para óleos vegetais, gorduras vegetais e creme vegetal. Diário Oficial [da] República Federativa do Brasil, 2005.

CODEX. Codex Stan 210, 1999. Codex standards for named vegetable oils. Codex Alimentarius, Rome, 2015.

Dean, L. L., Hendrix, K. W., Holbrook, C. C., \& Sanders, T. H. (2009). Content of some nutrients in the core of the core of the peanut germplasm collection. Peanut Science, 36, 104-120.

De Barros, I.A. \& Rodrigues, C. G. (2015) Avaliação da eficiência do óleo de amendoim na flotação de fosfatos. $e$-xacta, 8(2), 25-44.

EMBRAPA (Brasil). Embrapa algodão. Regiões produtoras de amendoim no Brasil. Sistemas de Produção, n. 7, 2. ed, versão eletrônica, 2014.

Gusso, A., Formaggio, A.R., Rizzi, R., Adami, M., Theodor, B.F. (2012) Soybean crop area estimation by modis/evi data. Pesquisa agropecuária brasileira, 47(3), 425-435.

Jorge, N.\& Luzia, D. M. M. (2012) Caracterização do óleo das sementes de Pachira aquatica Aublet para aproveitamento alimentar. Acta Amazonica, 42 (1), $149-156$.

Ma, Y., Kerr, W. L., Swanson, R. B., Hargrove, J. L., \& Pegg, R. B. (2014) Peanut skins-fortified peanut butters: Effect of processing on the phenolics content, fibre content and antioxidant activity. Food chemistry, 145, 883-891.

Moretto, E. Fett R.; Gonzaga, L.V.; Kuskoski, E.M. Introdução à ciência de alimentos. Editora da UFSC, 255 p., 2002.

Nunes, S. P. Produção e consumo de óleos vegetais no Brasil. Departamento de Estudos Sócio-Econômico Rurais, $159,2007$.

Oladipo, B \& Betiku, E. (2019) Process optimization of solvent extraction of seed oil from Moringa oleifera: An appraisal of quantitative and qualitative process variables on oil quality using D-optimal desig. Biocatal. Agric. Biotechnol., 20, 101187.

Pereira et al (2018). Methodology of cientific research. [e-Book]. Santa Maria City. UAB / NTE / UFSM Editors. https://repositorio.ufsm.br/bitstream/handle/1/15824/Lic_Computacao_Metodologia-Pesquisa-Cientifica.pdf?sequence=1.

PighinellI et al. (2008) Otimização da prensagem a frio de grãos de amendoim em prensa contínua tipo expeller. Ciênc. Tecnol. Aliment., 28, 66-71.

Pristouri, G., Badeka, A., Kontominas, M. G. (2010) Effect of packaging material headspace, oxygen and light transmission, temperature and storage time on quality characteristics of extra virgin olive oil. Food Control, 21(4), 412-418.

Radünz, M. et al. (2018) Avaliação de parâmetros de qualidade de óleos exóticos. Revista da Jornada de Pós-Graduação e Pesquisa-Congrega Urcamp, p. 791-800.

Shasidhar, Y., Vishwakarma, M. K., Pandey, M. K., Janila, P., Variath, M. T., Manohar, S. S., Varshney, R. K. (2017) Molecular mapping of oil content and fatty acids using dense genetic maps in groundnut (Arachis hypogaea L). Frontiers in plant science, 8, $794-804$.

Stevanato, N., Silva, C. (2019) Radish seed oil: Ultrasound-assisted extraction using ethanol as solvent and assessment of its potential for ester production. Industrial Crops and Products. 132, 283-291.

Tan, C. H., Ariffin, A. A., Ghazali, H. M., Tan, C. P., Kuntom, A., Choo, A. C. (2017) Changes in oxidation indices and minor components of low free fatty acid and freshly extracted crude palm oils under two different storage conditions. Journal of Food Science and Technology, 54 (7), $1757-1764$.

Win et al. (2011) Phenolic compounds and antioxidant activity of peanut's skin, hull, raw kernel and roasted kernel flour. Pak. J. Bot, 43(3), 1635-1642.

Zahran, H. A.; Tewfeuk, H. Z. (2019) Physicochemical properties of new peanut (Arachis hypogaea L.) varieties. Oilseeds \& fats Crops and Lipids, 26 (19), 17. 